\title{
SLOW BEAM EXTRACTION FROM THE NUCLOTRON
}

\author{
N.N.Agapov, V.A.Andreev, A.M.Baldin, N.A.Blinov, O.I.Brovko, V.N.Buldakovsky, \\ A.V.Butenko, O.M.Golubitsky, A.I.Govorov, E.V.Ivanov, I.B.Issinsky, S.V.Kalenov, V.I.Kaplin, \\ H.G.Khodgibagiyan, A.E.Kirichenko, A.G.Kochurov, A.D.Kovalenko, O.S.Kozlov, I.I.Kulikov, \\ L.A.Leonov, A.I.Malakhov, E.A.Matyshevsky, I.N.Meshkov, V.A.Mikhailov, V.A.Monchinsky, \\ P.I.Nikitaev, S.A.Novikov, S.V.Ronamov, P.A.Rukoyatkin, S.Z.Sayfulin, A.A.Smirnov, \\ B.N.Sveshnikov, V.V.Seleznev, B.V.Vasilishin, M.A.Voevodin, V.I.Volkov, A.V.Vol'nov. \\ LHE, JINR, Dubna, 141980, Russia
}

\begin{abstract}
Experiments on beam extraction from the Nuclotron, a superferric heavy ion synchrotron, were carried out last year and in March, 2001. The resonance Qx=20/3, used for a horizontal betatron amplitude growth, provides beam entering the Electrostatic Septum deflector, where it obtains an initial deflection to avoid losses on the septum of the Lambertson Magnet. The latter bends the beam in the vertical plane to the level of the experimental halls. An extraction coefficient of about $70 \%$ and a spill time of $0.4 \mathrm{~s}$ were obtained in the first experiments performed at the deuteron beam energy from 200MeV/amu to $1.5 \mathrm{GeV} / \mathrm{amu}$. Then, experiments on irradiation with $\mathrm{C}^{+6}$ and $\mathrm{Mg}^{+12}$ beams were performed on the external targets. The work, which permits one to improve the parameters of the extracted beam up to the projected ones, is discussed.
\end{abstract}

\section{INTRODUCTION}

The Nuclotron [1], a 6GeV/amu superconducting heavy ion synchrotron with iron shaped magnets, has been under operation since 1994, and physics experiments were carried out only on an internal circulating beam before March, 2000. The preparation of extraction equipment for final bench tests and installation in the ring started early in 1999. Extraction equipment assembling required the reconstruction of the regular dipoles and quadrupoles in the area of the 5-th, extraction, straight section. Last year the works were completed, and the equipment was installed into the ring. This allowed one to start the commissioning of the Slow Extraction System [2].

\section{EXTRACTION SYSTEM}

Two pairs of sextupole lenses are used to produce the 20-th harmonic of quadratic nonlinearity to excite the resonance $Q_{x}=20 / 3$. The first pair is located in the 2-nd and 6-th octants and the second pair in the 4-th and 8-th octants. The lenses of each pair connected in series are located at antipode points of the ring and supplied from separated sources. One pair is turned relative to the other one at an angle of $7 \pi / 16$. It allows one to have an arbitrary phase of sextupole disturbance.
Four additional quadrupole lenses $\mathrm{Q}_{\mathrm{ex}}$ supplied in series are used to reach the resonance frequency and to control the extraction process. The lenses are located in the 1-st, 3-d, 5-th and 7-th octants. The beam spill rate defining the extraction duration is controlled by a current slope, di/dt, in these lenses.

A blown up part of the beam is separated and deflected by the bending system [3] located in the 5-th straight section. Its first device, an Electrostatic Septum deflector, ESS, delivering a tension up to $200 \mathrm{kV}$ is placed in the first half of the section. The septum separating the region of the circulating beam from the region of the deflecting electric field is made of tungstenrhenium wires $0.1 \mathrm{~mm}$ thick. The beam in the gap is deflected outside the orbit in the horizontal plane at an angle of $2 \mathrm{mrad}$ to avoid losses on the iron septum of the Lambertson magnet, LM, located in the second half of the section.

The LM containing two $1.5 \mathrm{~m}$ sections bends up the separated beam in the vertical plane at an angle of $96 \mathrm{mrad}$ to the experimental halls located at the Synchrophasotron level. The LM superconducting windings are connected in series with the ones of the regular dipoles. This permits one to provide a constant angle permanently at any time of the magnetic ramp. The idea to compensate the LM leakage field in the gap for a circulating beam by means of the superconducting short-connected coil, proposed in [3], was successfully confirmed. The bench measurements of the field inside the gap have shown the field compression to a negligible value.

According to the process simulation $\mathrm{R}_{0}+19 \mathrm{~mm}$ should be an optimum radial operational position of the ESS and ML (the full aperture is $\pm 55 \mathrm{~mm}$ here). That is why in the region of the 5-th straight section it is necessary to have a horizontal orbit bump at the beginning of the cycle to meet requirements for unhampered beam injection and acceleration. Meanwhile, non-operational positions $\left(\mathrm{R}_{0}+63 \mathrm{~mm}\right)$ of the ESS and LM are foreseen in experiments with a fully free aperture. The devices are movable both in warm and cold states on keeping an ultra-high vacuum inside the beam chamber. This also allows the bending system to be fitted to the orbit position. 


\section{EXPERIMENTAL RESULTS}

According to our estimations, the most acceptable working points lie in the vicinity of $\mathrm{Q}_{\mathrm{x}}=6.79$ and $\mathrm{Q}_{\mathrm{z}}=6.84$ or $\mathrm{Q}_{\mathrm{x}}=6.73$ and $\mathrm{Q}_{z}=6.89$ (Fig.1). The approach to the resonance begins with a current change in the regular quadrupoles. The subsequent slow introducing current into the resonance quadrupoles permits one to move $\mathrm{Q}_{\mathrm{x}}$ to the value of $20 / 3$ providing a smooth control of the beam spill process (line segment 1-2, Fig.2).

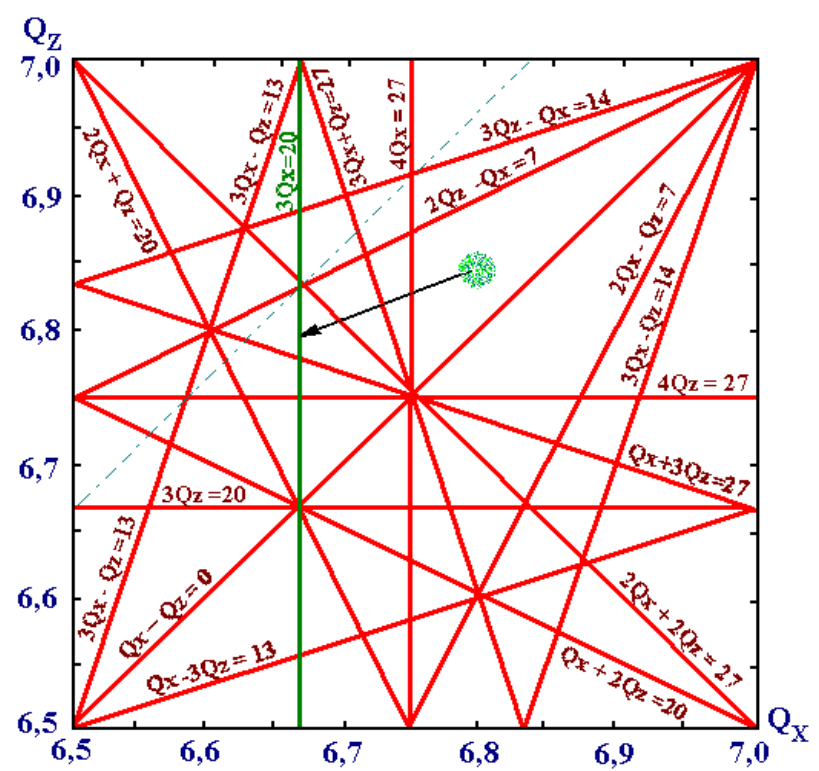

Figure 1: Resonance diagram near the working point.

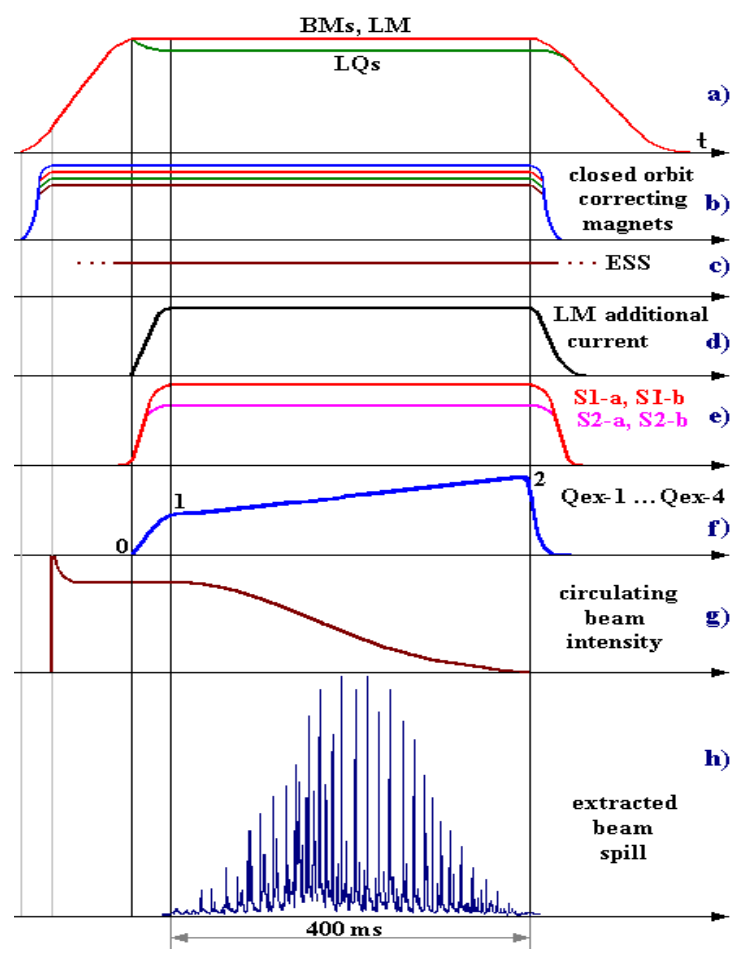

Figure 2:Timing diagram of the extraction processes.
Experiments on the amplitude growth at the resonance for the deuteron beam were performed before the installation of the bending system in the ring. The main magnetic flat-top field was $0.2 \mathrm{~T}$ corresponding to a particle energy of 204MeV/amu. A fluorescent TV-screen used to observe the blown-up beam was placed in more accessible warm straight section number 7 at a distance of $+20 \mathrm{~mm}$ from the central orbit. The movable screen permitted the aperture to be released during beam injection and acceleration. The observation of the resonance growth of the betatron amplitude resulting in hitting a beam unstable part on the TV-screen (Fig.3) has confirmed the capacity of the Resonance System operation.

\section{BEAM MONIT OR $1 \mathrm{CH1} \quad 22: 37$ 04.1298}

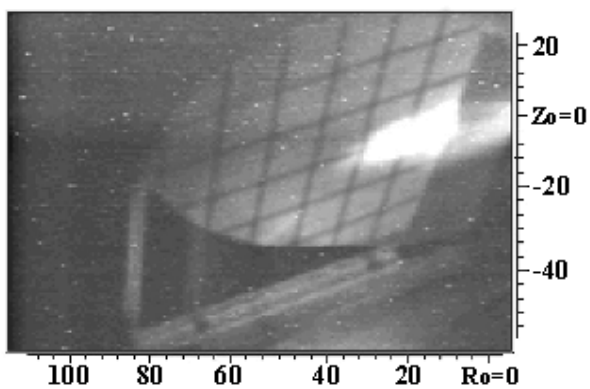

Figure 3: Image of the beam on the fluorescent screen.

The first extraction run began in the March of 2000 when the bending system was installed in the ring and the line was prepared for transporting the beam to the nearest external setup. A layout of the extracted system and beam position in the fifth straight section is shown in Fig.4.

The obtained typical beam extraction process, corresponding to an energy of $1.5 \mathrm{GeV} / \mathrm{amu}$ and a deuteron beam intensity of $10^{10} \mathrm{ppp}$ in Fig.2, illustrates the extracted beam spill for relevant currents. Apparent considerable time inhomogeneity of the beam is mainly due to current ripples in the lattice and extraction lenses, supplied by tentative sources.

The beam blown up in the horizontal plane and deflected in the ESS under operating conditions was observed on a fluorescent screen at the LM entrance (Fig.5a). Fig.5b shows the beam on a Polaroid film placed outside the ring vessel window.

In the March-2001run devoted to testing and tuning the Nuclotron, the laser ion source was used to accelerate carbon and magnesium nuclei. In these experiments, the beams of $3.10^{7} \mathrm{C}^{+6} \mathrm{ppp}$ and $2.10^{+5} \mathrm{Mg}^{+12} \mathrm{ppp}$ were extracted in the external channel where specimens were irradiated for an applied research group.

Measurements of the carbon beam extracted at an energy of $1.05 \mathrm{GeV} / \mathrm{amu}$ have shown that its emittance equal to $15 \pi \mathrm{mm} \cdot \mathrm{mrad}$ in the horizontal plane and $9 \pi \mathrm{mm} \cdot \mathrm{mrad}$ in the vertical one is in sufficient agreement with the calculated figures of the project. 


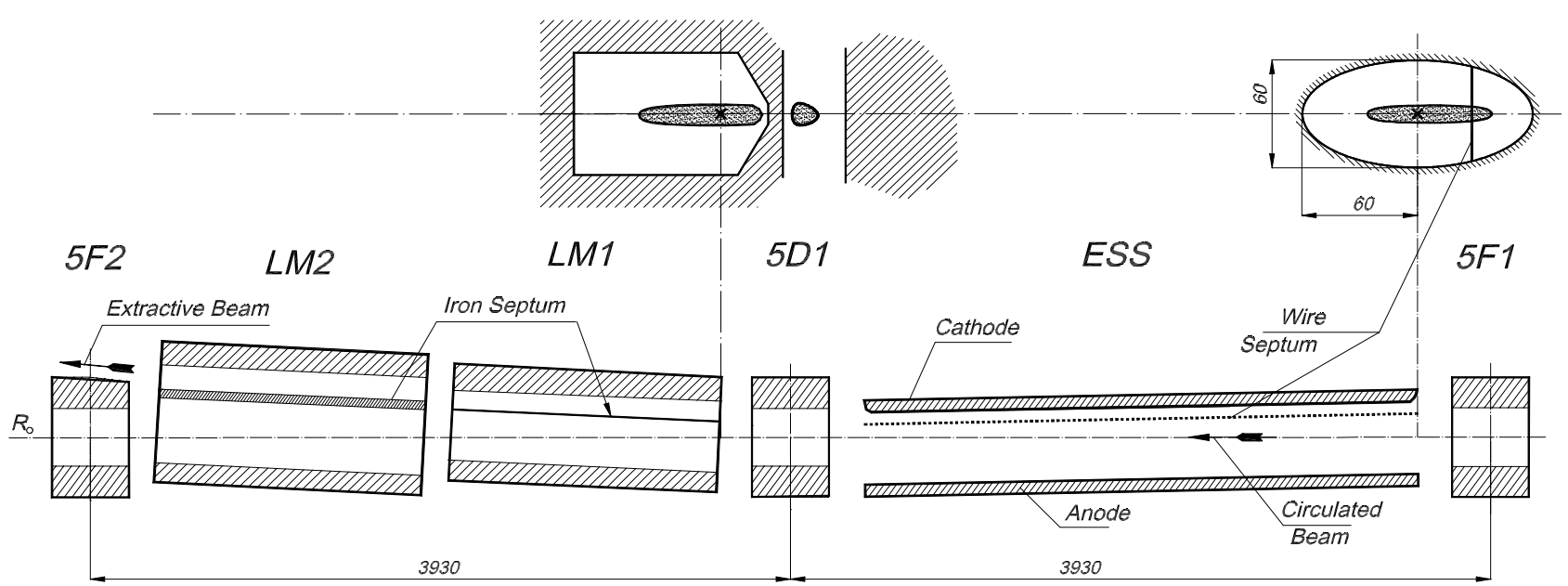

Figure 4: Layout of the extracted beam position in the fifth strait section (sizes in $\mathrm{mm}$ ).

a)

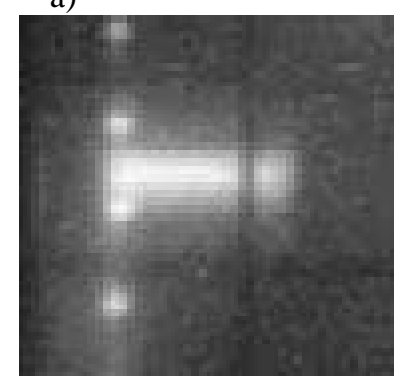

b)

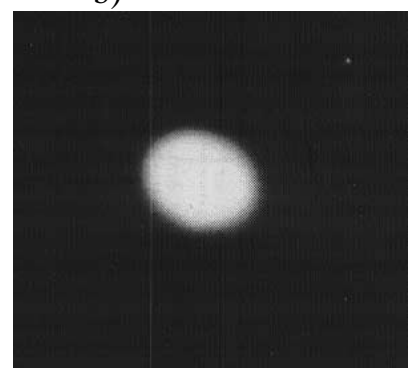

Figure 5: Beam image a) at the entrance of LM,

b) at the exit window.

Rough evaluations show that the extraction coefficient of the system over an energy range from $200 \mathrm{MeV} / \mathrm{amu}$ to $1.5 \mathrm{GeV} / \mathrm{amu}$ is about $70 \%$, though it should be much higher. It can be explained by not an accurate enough measurement of the beam intensity just before extraction or by the transition of the working point at its movement to $Q_{x}=20 / 3$ (Fig.1) close to the coupling resonance $2 \mathrm{Q}_{\mathrm{z}}-\mathrm{Q}_{\mathrm{x}}=7$ which could cause some beam losses.

Though the main task of the runs has been solved quite well, the obtained beam characteristics have not yet reached their projected values. The lack of time for tuning the extraction system was one of the reasons since we had to share it with the physicists who were pressing us by their requests to receive the external beam for their experiments.

\section{NEXT STEPS OF DEVELOPMENT}

To bring the characteristics of the external beam to the designed ones, such a kind of work has to be done:

- Increasing the accuracy in measuring the extraction coefficient. Investigation of the influence of the initial position of the working point on the extraction process and finding optimum ways of the approach to the resonance;

- Damping ripples in current supplies of the lattice and extraction lenses using, in consequence, feedback between a signal of the external beam and quadrupole current;

- Raising the extraction energy.

\section{ACKNOWLEGEMENT}

The authors greatly appreciate the contribution of many LHE employees to the implementation of the Nuclotron slow beam extraction.

\section{REFERENCES}

[1] A.Kovalenko.1994EPAC, Vol.1, p.161.

[2] I.Issinsky et.al.1997PAC, Vol.1, p.181.

[3] S.Averichev et.al.1996EPAC, Vol.3, p.2435. 